\title{
Eighth Korea-Japan Chemical Biology symposium: chemical biology notes from a small island
}

\author{
Tilman Schneider-Poetsch ${ }^{1}$, Shunji Takahashi ${ }^{2,3}$, Jae-Hyuk Jang ${ }^{4}$, Jong Seog $\mathrm{Ahn}^{4}$ and Hiroyuki Osada ${ }^{3}$
}

The Journal of Antibiotics (2016) 69, 885-888; doi:10.1038/ja.2016.58; published online 1 June 2016

\begin{abstract}
About 70 Scientists from Korea, Japan and Macao met in Naha, on the Pacific Islands of Okinawa for the Korea-Japan Chemical Biology symposium (KJCB), 18-21 January 2016. This marked the eighth meeting of the KJCB since the year 2000. In line with previous meetings, the beginning of the conference was somewhat hampered by inclement weather. Although moving the meeting closer to the equator was definitely a good idea, a good number of scientists spent part of the first meeting day amidst snow slush at Haneda airport in Tokyo waiting for a delayed takeoff. Hence, arriving at a balmy 17 degrees in Naha a few hours later proved quite a welcome relief.

The conference presented a fairly comprehensive overview of current research in chemical biology. Although the outline of the conference covered classical areas of research and methodology, beginning with discovery of bioactive molecules and continuing through biosynthesis before finally leading to mechanisms of active compounds, perhaps the most noteworthy aspect of the conference lay in connecting classical approaches with new technology, new methods, new targets and new uses.
\end{abstract}

\section{NEW APPROACHES TO SCREENING}

Testing thousands of molecules against a disease model has become a common way of seeking medically active molecules. However, screening experiments take place under artificial circumstances and do not truly model a disease in its host organism. In that respect, screening antimicrobial agents in silkworm larvae offers a more physiologically relevant screening environment. Hamamoto et al. ${ }^{1}$ of Tokyo University presented how Bombyx mori larvae infected with Streptococcus aureus allow a feasible in vivo system for new antibiotics. In terms of its pharmacodynamic and pharmacokinetic properties, the silkworm larvae appear to behave similarly to a mammalian organism. Although perhaps not suitable for the screening of tens of thousands of compounds, the silkworm system can accommodate several hundreds of molecules. In his presentation, Dr Hamamoto ${ }^{1}$ related how it allowed for the discovery of lysocin E (1) (Figure 1), which holds promising bactericidal activity.

\section{NEW SOURCES}

The risks associated with methicillin-resistant Staphylococcus aureus (MRSA) are well-known, and the need for antibiotics working against new targets is obvious. Ever since the discovery of the first antibiotics, scientists have scoured the earth to increase the supply of interesting molecules. Hence, the likelihood of finding something surprisingly new in the most easily accessible places is pretty low. Therefore, somewhat more exotic locations and sources of natural products are increasingly attracting attention. In this vein, the group of Jae-Hyuk Jang of the Korea Institute of Bioscience and Biotechnology. (KRIBB) has focused its attention on the volcanic island of Ulleung where they isolated strains of Catenulispora and Streptomyces. The bacteria produced various complex secondary metabolites including glycosylated triene macrolides and modified $\alpha, \beta$-dehydropipecolic acidcontaining cyclic depsipeptides named ulleungamides. ${ }^{2}$ The latter can only be correctly pronounced by native speakers of Korean. Ulleungamide A (2) displayed activity against S. aureus and Salmonella typhimurium.

Also targeting MRSA with new molecules, the group of Fukuda et al. ${ }^{3}$ of Kitasato University went to different places, coincidentally fairly close to the conference venue. Considering that water covers $70 \%$ of the earth's surface, marine life remains still underexplored as a source of natural products.

Working with fungal species isolated from Okinawan ocean sediment, Fukuda et al. ${ }^{3}$ isolated a family of thiodiketopiperazine metabolites named graphiumins (3), some of which proved toxic to S. aureus. As they appear to target production of staphyloxanthin, the bacteria's yellow pigment required for its virulence, Graphium holds the promise of acting upon a truly novel target.

Although ocean floor and volcanic island may appear still somewhat obvious choices for places to search for natural products, the lab of Dong-Chan Oh of Seoul National University searched for compounds in the symbiotic relationships between insects and microbes. Perhaps often overlooked, microbial symbionts have significant roles in the life and reproduction of insects. These include bacteria living in their digestive system. In contrast to above, the silkworms did not serve as a screening system, but their gut microbiota as producers of molecules

${ }^{1}$ Chemical Genetics Laboratory, RIKEN, Wako, Japan; ${ }^{2}$ Global Research Cluster, RIKEN-KRIBB Joint Research Unit, Wako, Japan; ${ }^{3}$ Chemical Biology Research Group, RIKEN Center for Sustainable Resource Science, Wako, Japan and ${ }^{4}$ Anticancer Agent Research Center, KRIBB, Cheongju, South Korea Correspondence: Dr H Osada, Chemical Biology Research Group, RIKEN Center for Sustainable Resource Science, 2-1 Hirosawa, Wako, Japan.

E-mail: hisyo@riken.jp

Received 16 March 2016; accepted 22 March 2016; published online 1 June 2016 


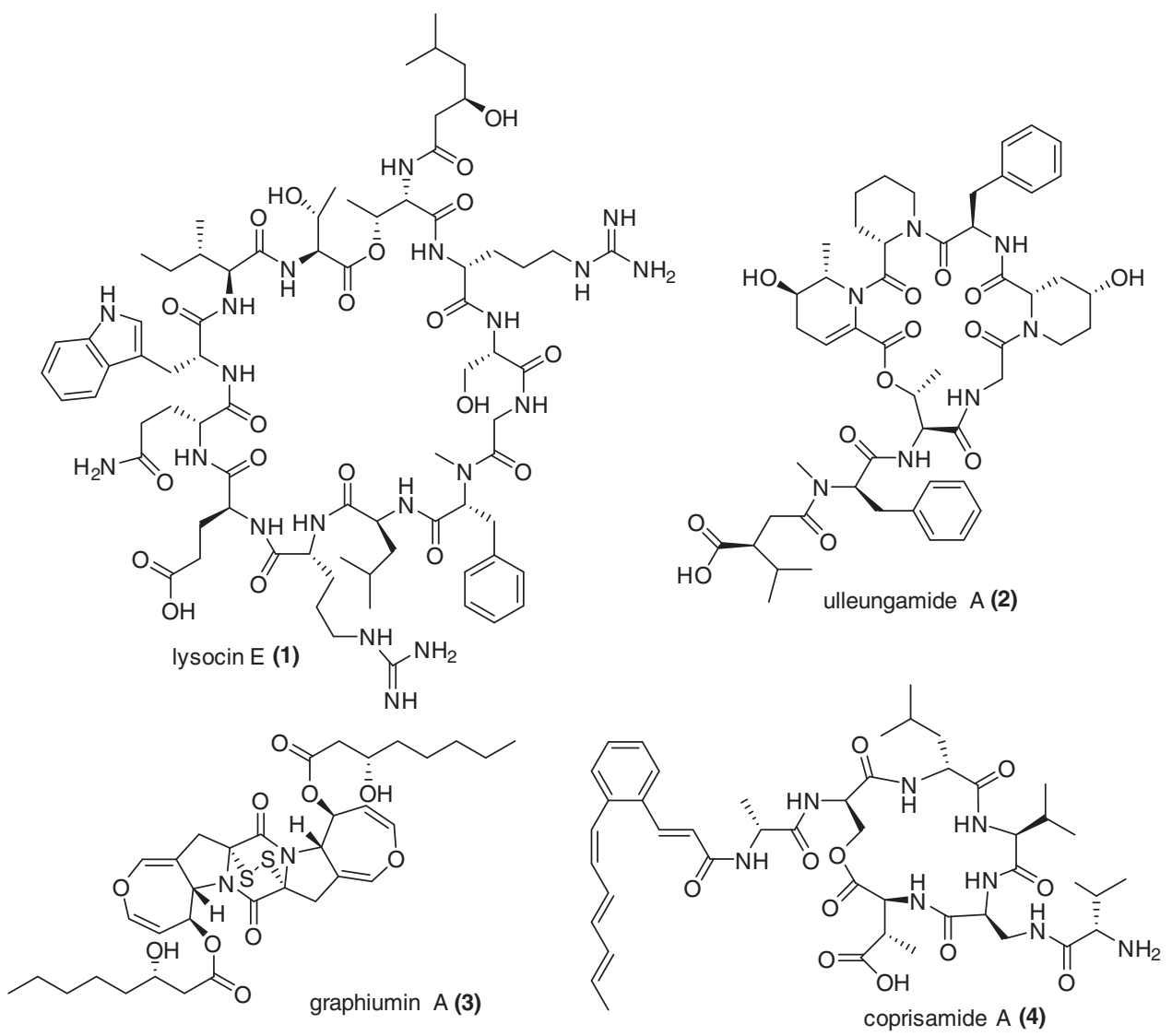

Figure 1 Novel compounds reported at this meeting.

worth screening. Secondary metabolites produced by the bacteria derived from silkworm's gut displayed significant activities against cancer cells and also to a limited extent against pathogenic microbes.

Interest in insect-associated microorganisms extends beyond the insect itself to the environment some insects create. Dung beetles (Copris tripartitus) are well-known, but few people have considered the microbiological environment inside dung balls a source of natural products.

Perhaps not the most attractive environment from the perspective of the researcher extracting molecules, the search uncovered coprisamides A (4) and B, two structurally unique cyclic peptides. ${ }^{4}$ Further new cyclic peptides were found associated with burying beetles (Nicrophorus concolor), an insect species that makes a living off decaying cadavers. A library of over 100 insect-associated unique molecules gives reason to further explore insect-associated microbiota for research and medical purposes.

\section{NEW SUPPLY OF OLD COMPOUNDS AND NEW COMPOUNDS FROM OLD MOLECULES}

The excitement over the discovery of novel molecules often overshadows the problem of isolating sufficient quantities of compound, and of maintaining a reliable source to ensure a steady supply of the discovered small molecule. By the time someone discovers interesting properties of a compound isolated years earlier, its source may have become unavailable.

Thus, the ability to engineer organisms to produce a desired molecule or at least precursors from which the compound of interest can be synthesized represents a useful and necessary discipline of chemical biology. Dairi and colleagues ${ }^{5}$ of Hokkaido University presented a particularly extreme example in which the original discovery of a molecule lay 40 years back. Cotylenin A, a molecule related to fusicoccin from the fungus Phomopsis amygdali induces apoptosis in cancer cells, however, the isolated fungal strain had lost its synthetic ability. The Dairi group succeeded in identifying the necessary machinery, including an essential P450 hydroxylase to allow production of an intermediate product.

Besides producing precursors for chemical synthesis, in vivo modification of small molecules also garners interest. Park et al. ${ }^{6}$ of Korea University presented how glycosylation changes the behavior and activity of various small molecules, including an increase in the bioavailability of tamoxifen.

Changing pharmacokinetic properties of known molecules also attracted the interest of the lab of Yoshimitsu Hamano at Fukui Prefectural University. The well-known antibiotic streptothricin displays little selectivity between eukaryotic and prokaryotic cells and is therefore unsuitable for clinical use. With the goal of engineering prokaryote-selective molecules, the Hamano group elucidated parts of the biosynthetic machinery responsible for chemical modification of the parent compound. ${ }^{7}$ Changing biological activity by modification of its biosynthesis presents one route of altering a putative drug's utility. However, how these changes will affect molecular behavior remains quite unpredictable. Therefore, modifying a molecule with predictable selectivity represents an often elusive goal in natural products research. A promising approach lies in conjugating molecules of known activity with other molecules that convey target specificity and selectivity. Kim and colleagues ${ }^{8}$ of Korea University presented advances and challenges in the field of siderophore conjugates. 
Siderophores, chelators used by bacteria for iron scavenging offer an opportunity to target siderophore-conjugated molecules for uptake by bacterial cells. ${ }^{8}$ In case of acinetobactin, the delivery to Aceintobacter baumannii, this concept appears promising, though owing to the selectivity of siderophore transport into the bacterial cell, attempts to conjugate ciprofloxacin to a metal chelator have thus far not yielded workable results.

\section{NEW SCREENING ENVIRONMENTS}

The need to selectively target a molecule in a complex biological environment also stresses that, cells, whether host or pathogen, whether healthy or tumorigenic do not live in isolation. Rather the interaction with their environment influences disease progression and also the efficacy of pharmacological intervention. Kawada et al. ${ }^{9}$ of BIKAKEN (Institute for Microbial Chemistry) explained his findings on the interaction of cancer and stromal cells, based on the observation that stromal cells appear to have a growth-limiting effect on tumors. This suggests that stromal cells produce and perhaps secrete signaling molecules that slow tumor formation. Surprisingly, one identified negative growth regulator turned out to be glyceraldehyde 3-phopsphate dehydrogenase $(\mathrm{GAPDH})$. Although its catalytic activity appeared unrelated, GAPDH seemed to accumulate on the cell surface in the vicinity of E-cadherin, and inhibit signaling via the mTOR and p70-S6K signaling pathways.

The example of GAPDH shows how entities, previously believed to be understood, can display surprising behavior. In contrast to a well-known enzyme having additional activity, one also has to remain cautious that the molecular mechanism of known compounds may turn out quite different than previously thought. Shim et al. ${ }^{10}$ of the University of Macau rectified former notions on the activity of nelfinavir. Originally developed as an human immunodeficiency virus (HIV) protease inhibitor, nelfinavir was thought to also inhibit the human $20 \mathrm{~S}$ proteasome and AKT (also known as protein kinase B) signaling. However, results appeared somewhat inconsistent, and a deeper investigation demonstrated that nelfinavir actually targets the chaperone Hsp90. As the HER2 receptor, notorious in certain types of breast cancer requires Hsp90 activity, nelfinavir displays strong selectivity for HER2-positive breast cancer cells.
In addition to molecules acting differently than previously thought and molecules isolated from exotic environs, the environment in which a clinical drug is applied naturally affects its efficacy. This may explain why treatment of tuberculosis requires 6-9 months of treatment as Mycobacterium tuberculosis can persist in a dormant state. Furthermore, most drug screens commence in a normoxic environment, which does not much resemble the conditions under which pathologic $M$. tuberculosis subsists. However, the $\mathrm{IC}_{50}$ for isoniazid increases $>10$-fold in a low oxygen environment.

Arai et al. ${ }^{11}$ of Osaka University introduced a screening system under hypoxic and low nutrient conditions, which they used to identify the marine sponge natural product agelasine D. Furthermore, overexpressing a mycobacterial transformant collection allowed for target identification via drug resistance.

Remaining on the topic of biology in low oxygen conditions, Nak-Kyun Soung of KRIBB presented a new way of pharmacologically targeting hypoxia-inducible factor alpha (hifl $\alpha$ ) using the natural product moracin-O. Moracin-O behaves in an unexpected manner as it does not target hifl $\alpha$ itself, but a novel regulator protein, which then suppresses hifl $\alpha$ expression.

The conference concluded with three presentations by young scientists.

Combining several disciplines within chemical biology, Asai et al. ${ }^{12}$ of Tohoku University used biosynthesis, chemical synthesis and pathway engineering to create an expanded range of polyketides. A cryptic polyketide pathway from Chaetomium indicum was heterologously expressed in Aspergillus oryzae grown under various growth conditions. Isolated compounds were chemically modified to increase variety while also attempting to reconstruct and engineer putative synthesis pathways.

In a similar vein, Yun et al. ${ }^{13}$ of RIKEN reported how activation of silent fungal synthesis pathways allowed production of tenuazonic acid and led to identification of the responsible biosynthetic enzyme tenuazonic acid synthetase 1 (TAS1), which combines polyketide and non-ribosomal peptide synthesis.

The symposium heavily focused on newly identified molecules, mechanisms and techniques. It became clear that besides finding new methods to harvest and modify molecules, comprehensive screening a

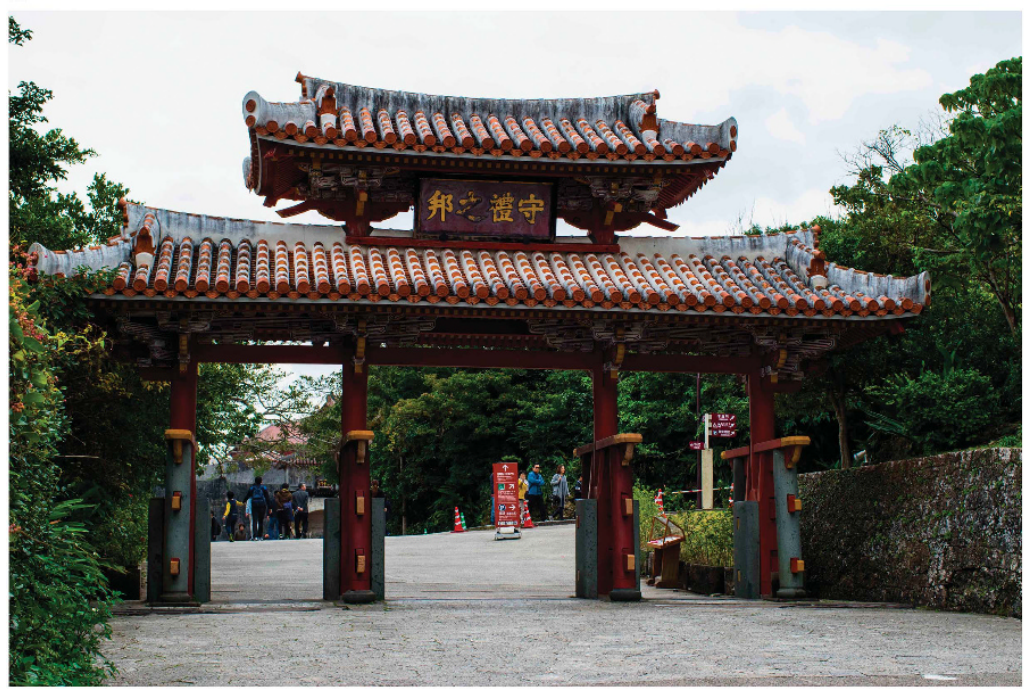

b

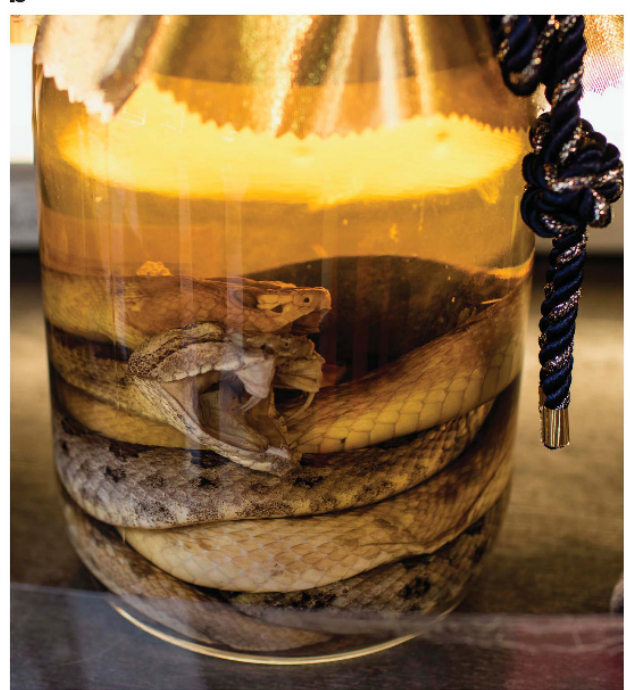

Figure 2 Impressions from the meeting venue. (a) Shureimon: entrance to the Shuri Castle, UNESCO (United Nations Educational, Scientific and Cultural Organization) World Heritage Site. (b) Habushu: a member of the pit viper family in $43 \%$ ethanol. 
under physiologically relevant conditions is of great importance to make the best use of chemical diversity. Hye-Jin Jung of Sun Moon University introduced three screening projects against melanoma, angiogenesis and glioblastoma, with particular emphasis on plantderived flavonoids. Her presentation stressed the use of compound that by themselves are not highly toxic, but may confer therapeutic benefits when combined with other anticancer drugs.

For a conference on Chemical Biology, Okinawa presents a very suitable venue. Not only did some of the molecules discussed originate from these very islands, but even among the Japanese, the denizens of Okinawa prefecture have the longest life expectancy. Part of this longevity is likely the result of the local cuisine. Although the conference did not cover chemoprevention in depth, the participants had sufficient opportunity to sample putative sources of beneficial small molecules at the final banquet and lunch, including various algae and bitter melons.

However, whether the intake of strong liquor in which poisonous snakes have been stored has any life-extending properties has not been conclusively determined. From the perspective of the snake, it clearly does not (Figure 2).

\section{CONFLICT OF INTEREST}

The authors declare no conflict of interest.
1 Hamamoto, $\mathrm{H}$. et al. Lysocin $\mathrm{E}$ is a new antibiotic that targets menaquinone in the bacterial membrane. Nat. Chem. Biol. 11, 127-133 (2015).

2 Son, S. et al. Ulleungamides A and B, modified alpha,beta-dehydropipecolic acid containing cyclic depsipeptides from Streptomyces sp. KCB13F003. Org. Lett. 17, 4046-4049 (2015)

3 Fukuda, T. et al. Graphiumins, new thiodiketopiperazines from the marine-derived fungus Graphium sp. OPMF00224. J. Antibiot. (Tokyo) 68, 620-627 (2015).

$4 \mathrm{Um}$, S. et al. Coprisamides A and B, new branched cyclic peptides from a gut bacterium of the dung beetle Copris tripartitus. Org. Lett. 17, 1272-1275 (2015).

5 Noike, M. et al. Molecular breeding of a fungus producing a precursor diterpene suitable for semi-synthesis by dissection of the biosynthetic machinery. PLOS ONE 7 , e42090 (2012).

6 Park, J. W. et al. Discovery of parallel pathways of kanamycin biosynthesis allows antibiotic manipulation. Nat. Chem. Biol. 7, 843-852 (2011).

7 Maruyama, C. et al. A stand-alone adenylation domain forms amide bonds in streptothricin biosynthesis. Nat. Chem. Biol. 8, 791-797 (2012).

8 Kim, H. S., Song, W. Y. \& Kim, H. J. Development of a novel fluorescence probe capable of assessing the cytoplasmic entry of siderophore-based conjugates. Org. Biomol. Chem. 13, 73-76 (2015)

9 Kawada, M. et al. Stromal cells positively and negatively modulate the growth of cancer cells: stimulation via the PGE2-TNFalpha-IL-6 pathway and inhibition via secreted GAPDH-E-cadherin interaction. PLOS ONE 10, e0119415 (2015).

10 Shim, J. S. et al. Selective inhibition of HER2-positive breast cancer cells by the HIV protease inhibitor nelfinavir. J. Natl Cancer Inst. 104, 1576-1590 (2012).

11 Arai, M. et al. Identification of the target protein of agelasine D, a marine sponge diterpene alkaloid, as an anti-dormant mycobacterial substance. Chembiochem. 15, 117-123 (2014).

12 Asai, T. et al. Use of a biosynthetic intermediate to explore the chemical diversity of pseudo-natural fungal polyketides. Nat. Chem. 7, 737-743 (2015).

13 Yun, C. S., Motoyama, T. \& Osada, H. Biosynthesis of the mycotoxin tenuazonic acid by a fungal NRPS-PKS hybrid enzyme. Nat. Commun. 6, 8758 (2015). 